\title{
Place Avoidance Tasks as Tools in the Behavioral Neuroscience of Learning and Memory
}

\author{
A. STUCHLÍK ${ }^{1}$, T. PETRÁSEK ${ }^{1}$, I. PROKOPOVÁ ${ }^{1}$, K. HOLUBOVÁ ${ }^{1}$, H. HATALOVÁ ${ }^{1}$, \\ K. VALEŠ ${ }^{1}, \breve{S}$. KUBÍK ${ }^{1}$, C. DOCKERY ${ }^{2}$, M. WESIERSKA ${ }^{3}$ \\ ${ }^{1}$ Institute of Physiology, Academy of Sciences of the Czech Republic, Prague, Czech Republic, \\ ${ }^{2}$ Albstadt-Sigmaringen University of Applied Sciences, Faculty of Life Sciences, Sigmaringen, \\ Germany, ${ }^{3}$ Nencki Institute of Experimental Biology, Warsaw, Poland
}

Received May 9, 2013

Accepted July 4, 2013

\section{Summary}

Spatial navigation comprises a widely-studied complex of animal behaviors. Its study offers many methodological advantages over other approaches, enabling assessment of a variety of experimental questions and the possibility to compare the results across different species. Spatial navigation in laboratory animals is often considered a model of higher human cognitive functions including declarative memory. Almost fifteen years ago, a novel dry-arena task for rodents was designed in our laboratory, originally named the place avoidance task, and later a modification of this approach was established and called active place avoidance task. It employs a continuously rotating arena, upon which animals are trained to avoid a stable sector defined according to room-frame coordinates. This review describes the development of the place avoidance tasks, evaluates the cognitive processes associated with performance and explores the application of place avoidance in the testing of spatial learning after neuropharmacological, lesion and other experimental manipulations.
\end{abstract}

\section{Key words}

Spatial learning - Cognitive coordination - Navigation • Orientation • Place avoidance • Lesions • Neuropharmacology • Learning $\bullet$ Memory $\bullet$ Behavioral tasks

\section{Corresponding author}

Ales Stuchlik, Institute of Physiology AS CR, v.v.i., Videnska 1083, 14220 Prague 4, Czech Republic. Fax: +420 241062488. E-mail: stuchlik@biomed.cas.cz

\section{Origin of place avoidance tasks}

The place avoidance task originated from circular arenas used in electrophysiological studies of place cells. The original version of the paradigm was invented by André Antonio Fenton in the group of Jan Bures, at the Institute of Physiology of the Academy of Sciences in Prague, Czech Republic, and first reported in 1997 (Bures et al. 1997a). As it had become clear that the activity of place cells is influenced not only by the environment, but also by the activity performed by the subjects and their experience, the group began experimenting with place preference and place avoidance tasks on a dry arena. In the place avoidance task, rats actively searched for food pellets dropped onto the arena (a set-up often used in place cell studies; Muller 1996) and a to-be-avoided sector was present but not directly perceptible, i.e. it involved locale navigation (navigation to hidden goals; O'Keefe and Nadel 1978). Upon entrance into the to-be-avoided sector, animals received mild footshocks. The arena could also be rotated, with the shock sector defined either according to the arena surface (the "arena-frame") or to the room-based landmarks (the "room-frame").

The active place avoidance emerged as a particular variant of this setup (Bures et al. 1997a,b). In this configuration, the arena was constantly rotating, and the shock sector was defined within the room frame. The active place avoidance task was soon recognized as an especially promising paradigm for research of spatial

PHYSIOLOGICAL RESEARCH • ISSN 0862-8408 (print) • ISSN 1802-9973 (online)

(c) 2013 Institute of Physiology v.v.i., Academy of Sciences of the Czech Republic, Prague, Czech Republic

Fax+420 241062 164, e-mail: physres@biomed.cas.cz, www.biomed.cas.cz/physiolres 
cognition. Rapid learning and robust performance of the animals were noted in the initial experiments: rats were able to learn the position of the sector in one session, and under extinction conditions (with no shock) continued to avoid the to-be-avoided area for more than $30 \mathrm{~min}$ (Bures et al. 1997b). The task is a dry-land paradigm and unlike traditional active and passive avoidance tasks (such as the two-way shuttle box or the step-through and step-down tests), localization of the sector requires place navigation abilities (Bures et al. 1997a), whereas execution of the avoidance reaction engages instrumental responses (Konorski 1948).

The active place avoidance task and other variants of place avoidance have therefore begun to be applied beyond place cells research, and has been recently employed in neuropharmacological (Stuchlik and Vales 2005, Vales and Stuchlik 2005, Stuchlik et al. 2008, Prokopova et al. 2012), lesion (Svoboda et al. 2008, Wesierska et al. 2005) and electrophysiological studies (Kelemen and Fenton 2010). The abovementioned experiments contributed to the development of the active place avoidance task, often referred to as the "active allothetic place avoidance" (AAPA) task (Stuchlik et al. 2004, Stuchlik and Vales 2005, Vales et al. 2006). Although this particular setup is the most commonly used, the place avoidance hardware (sometimes called "the Carousel Maze", Prokopova et al. 2012, Vales et al. 2012) can be easily adapted for other related tasks, providing a useful and flexible tool for answering specific experimental questions.

\section{Principle of the active place avoidance}

In principle, the active place avoidance represents a simplified and specialized place avoidance task, in which the shock sector is fixed according to room-frame coordinates. The apparatus used for the active place avoidance is schematically shown in Figure 1. An animal is placed on a slowly rotating arena and trained to avoid a stable sector defined by its spatial relationships to distal room cues. Since the task is active by nature and requires ongoing locomotion, animals need not be motivated to forage for food pellets, although sometimes the motivation and foraging are used, especially when other passive versions of place avoidance are employed (Wesierska et al. 2005).

The great advantage of the task is the very rapid acquisition, enabling to assess even short-term changes in performance of animals such as during the estrous cycle
(Cimadevilla et al. 2000a). Since active place avoidance is a dry-arena task, it does not require swimming and thus can be used for weanling rats just as for adults (Cimadevilla et al. 2001a). It can be used even for testing mice. For mice, the active place avoidance was modified so that the electric shock was replaced with an air puff from several jets mounted equidistantly on the arena periphery (Cimadevilla et al. 2001b). Moreover, mice can be trained in the task using an electrified grid floor (Burghardt et al. 2012).

The active place avoidance has several remarkable aspects. The to-be-avoided sector is defined solely by its room-frame coordinates and the animals thus cannot learn the task by simple associative learning; instead they probably use allothetic (Bures et al. 1997a,b, Cimadevilla et al. 2000b) strategy. The idiothetic mode of navigation (based on the collection and integration of self-motion signals, Stuchlik et al. 2001) and substratal exteroceptive cues (such as scent marks), are irrelevant and animals must ignore them, because they "rotate with the arena", whilst the sector is stable.

The optimal strategy to avoid punishment is to recognize the position of the shock sector and stay away from it by periodically walking in the direction opposite to the rotation. Simple suppression of locomotion is a very inefficient strategy in this task (as opposed to passive avoidance paradigms), since the arena rotation repeatedly transports the rat into the sector if it does not actively avoid it. Although an allothetic strategy is crucial for efficient performance, alternative non-spatial strategies might also play a role in solving the task. It is conceivable that a purely locomotor or procedural strategy, in which animals simply walk at a constant velocity counter-rotation without any spatial awareness, might provide solutions to the task. An unpublished experiment by our laboratory addressed this issue by training rats in darkness to test if rats are able to solve the active place avoidance by employing a purely procedural strategy (possibly involving interval timing) (Buhusi and Meck 2005) in the absence of distal cues. The rats were only able to perform the task in darkness if they had previous experience with the task in the light, i.e. they were familiar with the task rules. Even so, avoidance in darkness was less efficient than in the light. Naive animals, introduced directly to the dark arena, were not able to perform the task at all. This suggests that a purely locomotor skill is not sufficient for efficient performance (Klement, Kenney, Fajnerova; unpublished data).

The active place avoidance allows for 


\section{Schematic diagram of the place avoidance setup}

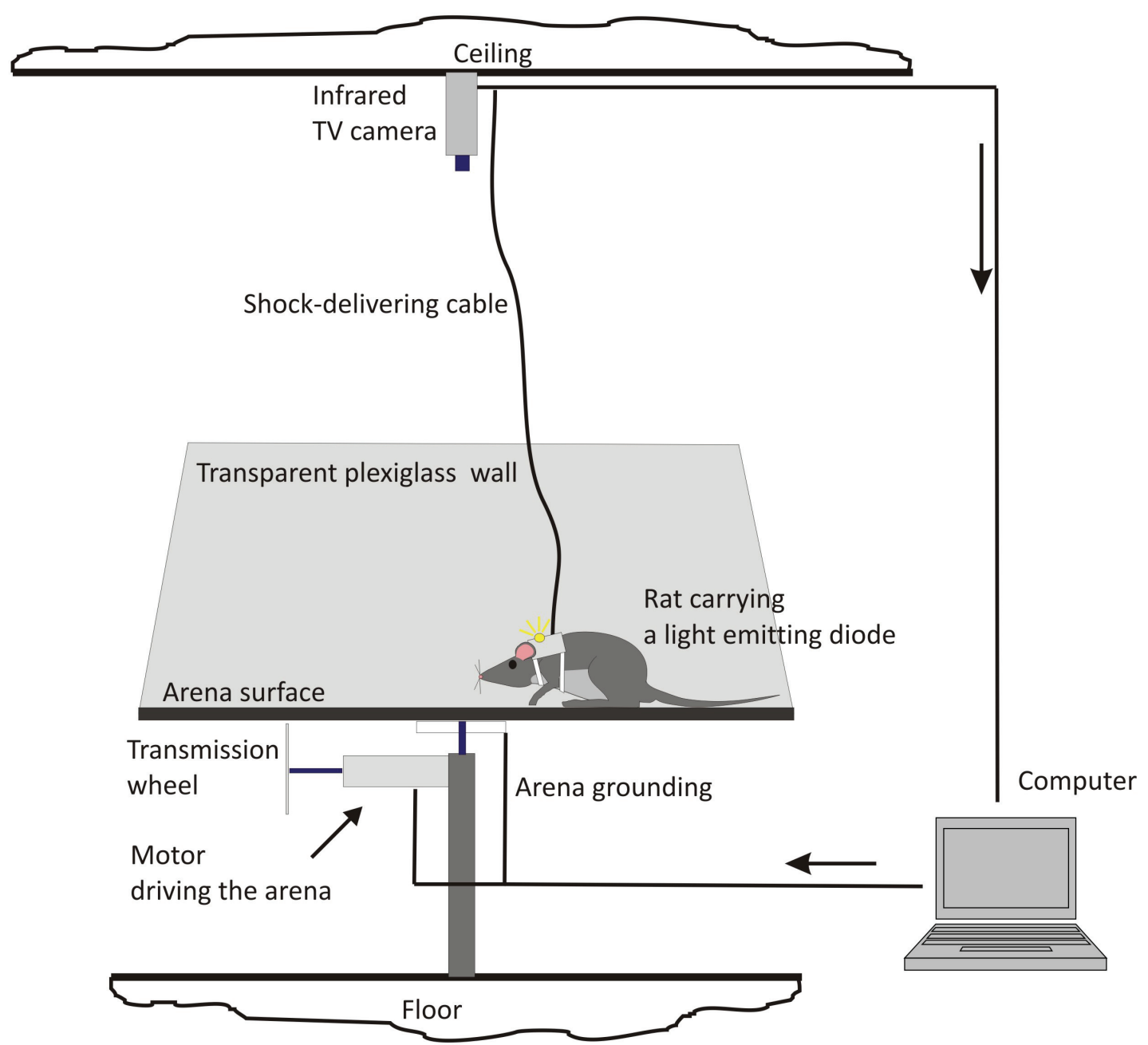

Fig. 1. Schematic drawing of the place avoidance setup. A smooth, metallic circular arena (the "Carousel Maze") is located in the experimental room containing numerous extramaze cues. Rats walk on the grounded arena surface, and upon entrance into the to-beavoided sector, they receive mild footshocks delivered from the shock cable which is connected to the computer-driven constant-current generator. The computer based tracking system (iTrack; Tracker, Biosignal Group, USA) records the position of the animal every 40 ms in the coordinate frames of both the room and the arena (the latter reflecting active locomotion only). Animals wear a small latex jacket fitted with an infrared light-emitting diode (LED) which is registered by a TV camera mounted on the ceiling.

investigation of cognitive coordination (see later), in addition to spatial learning. It also requires ongoing locomotor activity and the animal's attention to room cues. We propose that spatial mapping (representational) demands in the place avoidance are lower than in the Morris water maze (MWM; Morris 1981) or Barnes maze (Barnes 1979), because animals avoid a relatively large part of the environment. However, cognitive coordination makes the task difficult and susceptible to bilateral and unilateral hippocampus inactivation with tetrodotoxin (TTX; see later). Testing of cognitive coordination is highly relevant for animal models of psychiatric disorders, such as schizophrenia-like behavior in rodents (Stuchlik et al. 2004, Stuchlik and Vales 2005,
Bubenikova-Valesova et al. 2008). Caution must be paid to attain the optimal level of negative reinforcement, i.e. careful titration of the shock current; as both too low and too high intensity of the footshock can compromise an animal's motivation or ability to learn and perform the task.

\section{Parameters measured in the place avoidance tasks}

The active place avoidance and commerciallyavailable analysis program (Track Analysis, Biosignal Group, USA) provide a rich set of parameters. Here we discuss only the most commonly used ones, although 
other parameters may be chosen optionally to address more specific experimental questions.

A primary measure is the number of entrances into the to-be-avoided sector (or the number of errors) within a given session. It is a measure of the cumulative performance in the task. The maximum time avoided (or maximum time between two errors) reflects the ability to maintain avoidance throughout a session; these two parameters correlate highly in general. The time to the first error is the latency to the first entrance into the sector in a given session. While keeping the sector position constant across experimental days it is essentially a measure of between-session learning, i.e. retrieval of memory from the previous session. If a rat fails to escape from the sector, shocks are repeated at regular intervals (usually every $1200 \mathrm{~ms}$ ). A high number of shocks per entrance may indicate that the rat is not able to escape properly. This occurs more often at the onset of learning, when the animal has not yet understood the different variables involved in the task (e.g. the shock sector location and size, the repetitive nature of the shock). This may also occur due to impaired spatial association, decreased shock sensitivity, low shock intensity, motivational deficit or also due to motor or cognitive impairment. A low ratio of shocks/entrance indicates that the rat successfully adopted skills to escape and/or avoid, and has been used as an index of cognitive skill learning (Dockery and Wesierska 2010). To control for the possible confounding effect of a motor impairment, it is important to monitor the active locomotion of the animals. The total path length usually refers to the length of the arena-frame path during an entire session. In the case of ataxia or severe hypolocomotion, the numbers of errors as well as other avoidance parameters cannot be related to spatial performance and the degree of cognitive dysfunction per se and rather explicitly reflect motor disability. Another reason for decreased locomotion might be excessive anxiety-related freezing behavior, which prevents learning of the task (Carr et al. 2011). The occurrence of such "poor learners" can be reduced by using appropriate shock levels and habituation of the animals to the apparatus.

\section{Specific cognitive functions assessed by place avoidance tasks}

Experiments in the original place avoidance tasks provided useful insights about the nature of the cognitive load in the active place avoidance. It is widely recognized that spatial navigation consists of two largely independent functions: allothetic navigation - using distant landmarks and their relationships, and idiothetic localization - using information generated by the animal itself, such as vestibular, motor efference, and proprioceptive information. Those functions might be differentially affected by various manipulations (drugs, brain lesions, etc.). Behavioral and later electrophysiological experiments involving both the stable and rotating arenas have shown that independent spatial memory representations may be encoded by neuronal coalitions in an alternating fashion in the arena and room reference frames (Kelemen and Fenton 2010; see later).

Original studies using place avoidance variants: dissociation of spatial reference frames

The initial studies on place avoidance (Bures et al. 1997a,b) presented several intriguing experiments involving navigation in the arena- and room-frames. One group of rats was trained in the place avoidance task on a stable arena in light. Here, rats could associate the position of the to-be avoided sector with both local and distant cues including idiothetic input. During extinction (with the shock inactivated) in darkness, the rats continued to avoid for tens of minutes, using substratal cues on the arena surface and idiothesis until avoidance was eventually extinguished. When the lights were turned on again, the rats continued to avoid, but then only according to the room-frame defined sector (as revealed, when the arena was rotated). This is explainable by an independent extinction of the arena-frame avoidance on the rotating arena in darkness. The room-frame memory, however, remained intact and was recalled when room cues became available again. A second group of rats was initially trained on a rotating arena in light with the to-beavoided sector defined solely according to the roomframe. These rats could not learn arena-based avoidance, because local cues were made irrelevant. Accordingly, they showed no signs of avoidance during the extinction session in darkness, when the room-frame information was unavailable. Animals resumed the room-frame avoidance as soon as the light was turned on. This demonstrated that independent memories are formed autonomously for each of the two spatial reference frames and can be separately extinguished (Bures et al. 1998).

\section{Autonomous expression of arena- and room-frames}

An even more impressive demonstration of this principle was shown by Fenton et al. (1998). The task 
involved collecting food pellets on a stable arena in light with a single to-be-avoided sector. When in a later phase the arena was rotated, animals successfully avoided not only one, but two sectors at once; one frame being defined relative to the arena, and the other defined by allothetic room cues (Fenton et al. 1998). Rats thus acquired autonomous memories for both the arena- and room-frames. In this study, avoidance appeared during the extinction condition when shock was inactivated for both frames. A more conclusive picture of hippocampal place cell coding in the double (or two-frame) place avoidance tasks has recently been provided by Kelemen and Fenton (2010). They recorded activity of CA1 pyramidal neurons in rats solving the double (or twoframe) place avoidance task (with the shock activated), in which the rats had to avoid both sectors in one session. An analysis of the firing patterns showed that the neurons were divided into sub-populations of coactive cells, one of which encoded the position according to the room reference frame, and the other according to the arenaframe. Only one representation was active at any given time, preventing their superposition, but they alternated on a timescale of milliseconds to seconds. Such temporal separation of different representations was called dynamic grouping (Kelemen and Fenton 2010). Moreover, when the animal was close to one of the sectors, or entering it, the ensemble encoding the corresponding spatial frame was more likely to be active, suggesting that the more behaviorally relevant representation was active while the other one was suppressed. This is direct evidence of a neuronal manifestation of cognitive control, or cognitive coordination, which involves the process of distinguishing the relevant stream of information from the irrelevant, and choosing the optimal response.

\section{Segregation of stimuli into coherent subsets and cognitive coordination}

"Cognitive coordination" can be described as a process allowing the brain to selectively activate certain existing representations, while suppressing other unrelated representations, in order to employ a successful behavioral strategy (Phillips and Silverstein 2003, Wesierska et al. 2005). On a cellular level this can be understood as the timing of neuronal activity and the forming of coalitions of place cells with no changes in the character of activity of individual neurons or in the organization of their firing fields (Olypher et al. 2006).

The place avoidance tasks offer the opportunity to assess cognitive coordination in animals by bringing distal and proximal cues into direct conflict. Arrays including multiple variants of the place avoidance task, which differ in the availability and relevance of the reference frames, have been used to examine the presumed dominant role of the segregation of information from dissociated reference frames (Wesierska et al. 2005, Kubik and Fenton 2005). These versions were named according to the availability of information from the respective reference frame (Room or Arena) and its relevance to the task solution ( + or - sign). Specifically, the conflict between the reference frames may be eliminated in (Room\&Arena)+ avoidance on a stable arena in light where information from either frame yields congruent information (Fig. 2A). In the Arena+ avoidance (Fig. 2B), room-frame visual cues are hidden by darkness and the to-be avoided sector is defined relative to the rotating arena. In Room + avoidance, the arena is covered in shallow water $(\sim 1 \mathrm{~cm})$, suppressing substratal cues (e.g. olfactory) on the arena surface and thus attenuating the reference frame conflict (Fig. 2C). The idiothetic cues cannot be eliminated, but their capacity to support successful avoidance is limited (Stuchlik and Bures 2002). In this system, the active place avoidance on the dry rotating arena itself is referred to as the Room+ Arena- avoidance (Fig. 2D), reflecting the fact that both room and arena stimuli are present, but in conflict, with only the room frame providing relevant information (due to the to-be-avoided sector being defined according to the room-frame). Perhaps the most difficult variant of the place avoidance is double (twoframe) place avoidance (Fig. 2E), where a rat must avoid two sectors, one rotating with the arena, another stable in the room (Kelemen and Fenton 2010).

\section{Evidence for a hippocampal role in cognitive coordination}

Wesierska et al. (2005) used these different versions of place avoidance with foraging to study the specific effects of unilateral hippocampal inactivations on the place avoidance behavior in more detail. A series of experiments showed that the detrimental effect of unilateral infusion of TTX into one hippocampus was specific to the dissociation of reference frames. It was most prominent in the Room+Arena- avoidance (active place avoidance with foraging) (Fig. 2D), in which information from both reference frames was fully present and the conflict between the dissociated spatial reference frames was most pronounced. When this conflict was 


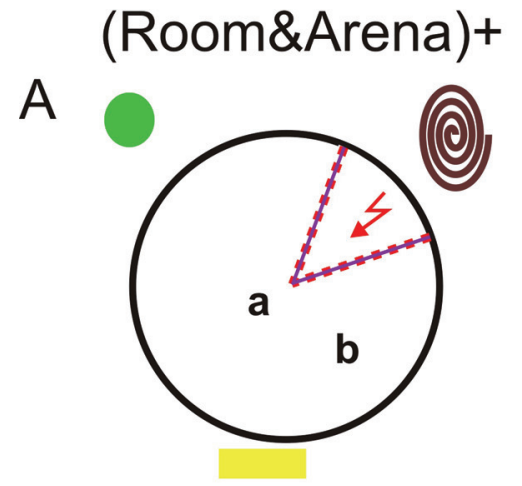

Arena stable in light

Room and Arena information relevant

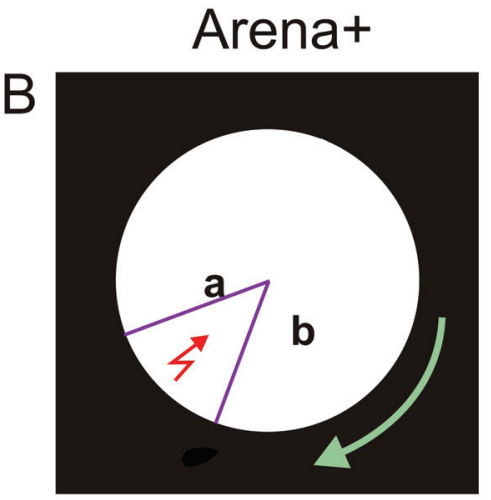

Arena rotating in darkness

Arena information relevant

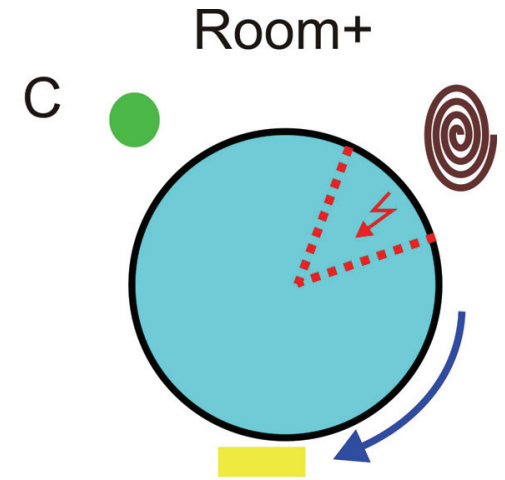

Arena with shallow water rotating in light

Room information relevant

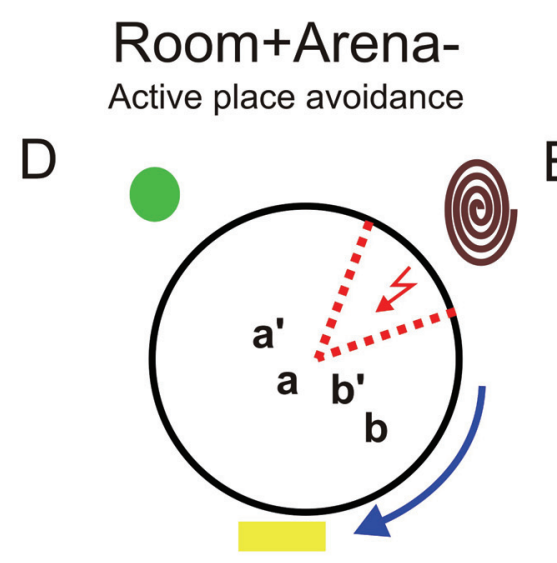

Arena rotating in light
Room+Arena+

Two-frame (double) avoidance

\section{E}

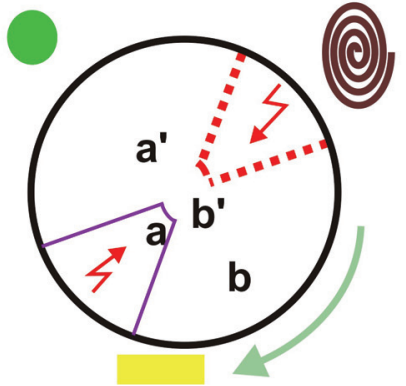

Arena rotating in light

Room information relevant Arena information relevant

Fig. 2. Schematic illustration of the place avoidance variants: Versions are denoted by information from the respective reference frame (Room or Arena) and its role in the performance (+ or - sign). A: (Room\&Arena)+ avoidance involves training on a stable arena in light where information from both frames defines a stable sector. There is no conflict of the frames and rats must be motivated to locomote by foraging for food pellets dropped regularly on the arena floor. B: Arena+ avoidance is conducted in darkness and the tobe avoided sector is defined relative to the rotating arena. Animals must again forage for food. C: In Room+ avoidance, the arena is covered by water $(\sim 1 \mathrm{~cm})$, suppressing substratal cues and thus attenuating the reference frame conflict. D: Room+Arenaavoidance (active place avoidance) has both room and arena stimuli present, but only the room stimuli are relevant as the sector is defined according to the room-frame. Here, arena-bound cues must be ignored. Rats may or may not collect food pellets. E: Room+Arena+, two-frame (double) avoidance. In this task, an animal is required to avoid two sectors - one defined with respect to the rotating arena, and the second according to the room-frame coordinates. Both sectors are truncated from the center in order to provide a space which allows for efficient escape responses. In this task animals forage for small food pellets. The geometrical figures presented outside the arena depict extramaze cues, whereas the letters on the arena depict intramaze cues.

absent, such as in the (Room\&Arena)+ avoidance on a stable arena in light (Fig. 2A) or in the Arena+ avoidance in darkness (Fig. 2B), performance was spared despite unilateral hippocampal blockade. Attenuating the conflict by covering the arena with shallow water (Room+ avoidance) (Fig. 2C), which suppressed arena-based stimuli, also partially spared the avoidance. These results suggested that animals with one hippocampus have normal spatial learning and motor abilities, but they are impaired in cognitive coordination. This function is used to solve the conflict between multiple discordant streams of information. These results suggest that the active place 


\section{Examples of rats' trajectories after experimental manipulations}
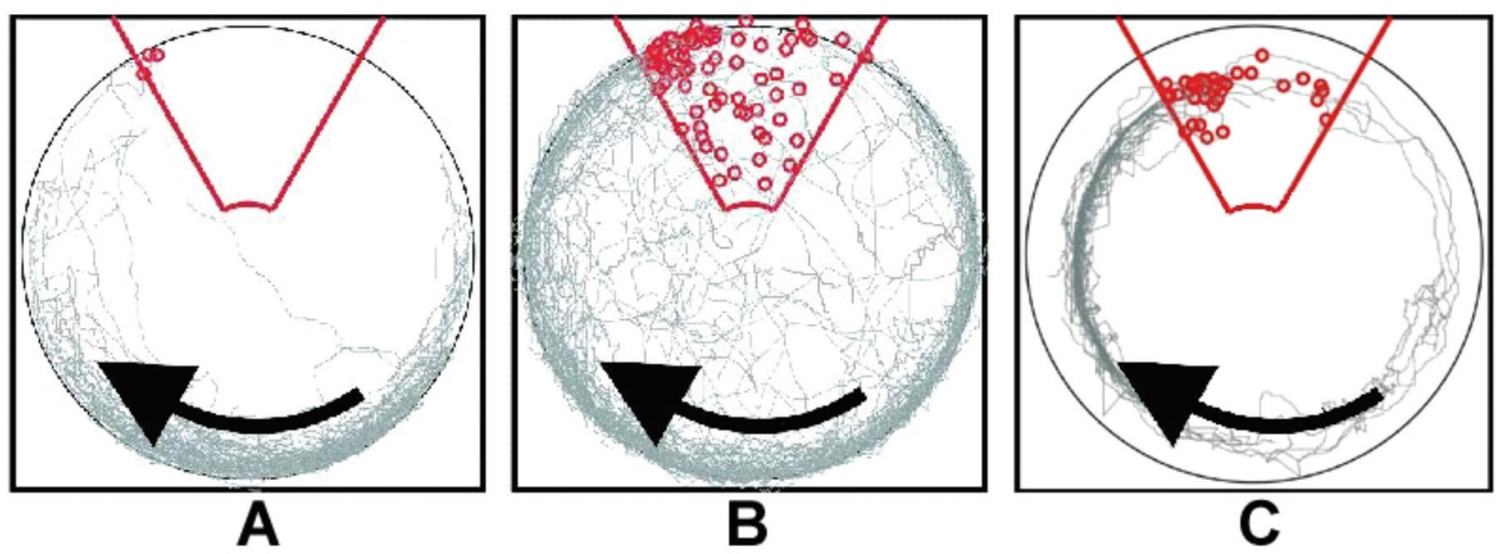

Fig. 3. Examples of representative trajectories of rats after selected pharmacological manipulations. Presented are the trajectories of A: a control animal injected with saline; the animal efficiently avoided the sector with locomotion with the activity concentrated on the safe part of the arena, B: a rat administered with $0.2 \mathrm{mg} / \mathrm{kg}$ of MK-801 (dizocilpine), a noncompetitive NMDA receptor antagonist (there is a severe avoidance deficit which is accompanied by pronounced hyperlocomotion.), and C: a rat administered with $25 \mathrm{mg} / \mathrm{kg}$ of propranolol, exhibiting an avoidance deficit, but still capable of escape reactions. The arrow indicates the direction of the arena rotation.

avoidance on the rotating arena in light requires segregation of spatial stimuli and coordination of behavior according to separate representations of the surrounding environment. In this regard, the hippocampus plays a key role in these cognitive processes.

The possibility to test cognitive coordination in animal models is very attractive since dysfunctions of this process are currently considered to be one of the symptoms of schizophrenia (Ellenbroek and Cools 1990, Phillips and Silverstein 2003). Schizophrenic patients have difficulties with differentiation between important and unimportant stimuli and with establishing associations between important stimuli. In some ways this resembles deficits observed in rats with an inactivated hippocampus (Olypher et al. 2006). Experiments comparing the differential effects of TTX infusion into one hippocampus on learning and retrieval in the Room+ avoidance variant (shallow water attenuating the reference frame conflict on a rotating arena in light) vs. a spatial water maze suggested that segregation of stimuli and the construction of spatial representations are distinct functions of the hippocampus, which are dissociable on the behavioral level (Kubik and Fenton 2005). In the Morris water maze, unilateral inactivation prevented retrieval of a previously learned spatial behavior, but not new spatial learning. Conversely, in the Room+ avoidance task, inactivation prevented new learning, but spared retrieval of previously learned avoidance. It appears that each of these tasks tests different hippocampal functions as they are clearly differentially affected by unilateral inactivation. Segregation of stimuli into coherent subsets is critical in the rotating arena with multiple reference frames, while no explicit reference frame conflict is present in the MWM.

Electrophysiological recordings by Lee et al. (2012) demonstrated that the degree of interhippocampal synchrony of local field potentials in beta, delta and theta oscillations exhibits strong correlation with performance in the active place avoidance task. The synchrony was disturbed in a neurodevelopmental animal model of schizophrenia, together with task performance. This observation further corroborates the view that cooperation between the two functional hippocampi is needed for cognitive coordination and thus mastering the active place avoidance.

\section{Task complexity, working memory and skill learning}

Another variant of the place avoidance task, the allothetic place avoidance alternation task (APAAT) is focused on visuospatial working memory. Moreover, the experimental design allows for loading of task difficulty (Dockery and Wesierska 2010). In the setting used by Dockery and Wesierska (2010), the sector position was changed between daily sessions (in a manner analogous to changing platform positions in the working memory version of the water maze). The shock sector, defined by room-frame coordinates (Room+Arena-), was narrower than in other variants 
$\left(45^{\circ}\right)$. In order for the previous sector location to be extinguished, rats began each session with a five-minute habituation (rotating arena, shock inactivated) before undergoing training for the new sector. After the habituation interval, within a session rats underwent two five-minute training intervals, a five-minute delay and a 5-min retrieval test with no shocks. Visuospatial working memory (Dockery and Wesierska 2010) was indexed by testing rats after a delay interval in which the animals were placed outside the arena. Across sessions the rats had three days of working memory training sessions. The long-term influence of previous exposure and training on skill learning can then be retested in a fourth session. In this particular study, rats were tested three weeks later with the shock sector again located in a novel position. The ability of the rats to avoid rather than escape the sector is an index of retention of the learned cognitive skill which is place avoidance itself (Dockery and Wesierka 2010).

\section{Reversal learning and mnemonic segregation}

A very simple and potent method of increasing the cognitive load of active place avoidance is to change the sector position by 180 degrees relative to that learned by the subjects during training, a condition that can be called simply "reversal" or "conflict learning" (Abdel Baki et al. 2009). Adjustment to this change requires a specific kind of cognitive flexibility, which has been described as "mnemonic segregation" (as opposed to spatial frames segregation) by Abdel Baki et al. (2009). The rats have to distinguish the old and irrelevant spatial representation from the new and relevant, while the need to segregate spatial frames remains. The ability to differentiate between similar memories and choose the appropriate behavior is probably dependent on hippocampal pattern separation (Burghardt et al. 2012).

\section{Proximal cues and idiothetic navigation in place avoidance}

Proximal cues (e.g. scent marks on the arena surface) and idiothesis (e.g. path integration) are generally harder to control and manipulate than distal landmarks. The rotating arena tasks easily allow dissociation of extramaze and intramaze inputs, but in typical spatial tasks one cannot discriminate the use of intra-arena cues from idiothesis. Idiothesis alone, without any other correcting spatial input, is not a reliable navigational strategy over long time intervals, because it accumulates translational and especially rotational errors. Therefore, we developed a twosegment arena which enabled constant shuffling of the substratal cues on the arena surface rendering the intraarena cues unreliable (Stuchlik et al. 2001, Stuchlik and Bures 2002). The arena consisted of a central disc (85 $\mathrm{cm}$ diameter), and an outer belt (25 $\mathrm{cm}$ width). The rat had to move from the disc to the belt and back in order to trigger delivery of food pellets. While the rat was on one part of the arena, the other was rotated by $60^{\circ}$ (Stuchlik and Bures 2002). Extramaze cues were hidden by darkness and, for some animals, were also made irrelevant by rotation of the whole apparatus. Surface shuffling did not affect performance on a stable arena, presumably due to the availability of residual extramaze cues, which were sufficient to correct the cumulative error in idiothetic navigation. On a rotating arena (whole apparatus rotating), with room-based cues rendered irrelevant, rats had to rely on path integration only during shuffling of the arena surface, and their performance declined sharply - they were able to navigate successfully on a path up to 5-7 m, until they committed an error. Another very straightforward method of devaluating proximal cues was used by Wesierska, Dockery and Fenton (2005). The arena was covered by a shallow $(\sim 1 \mathrm{~cm})$ layer of water, which at least partly suppressed intramaze cues such as scent marks.

\section{Importance of inertial stimuli}

A recent study (Blahna et al. 2011) tested the role of so-called "inertial" stimuli in place navigation in the active place avoidance. These inertial stimuli are generated both by the active locomotion of the animals together with the passive transport caused by the rotating arena. A modified version of the active place avoidance was developed, in which rats walked on the stable substrate and the surround (i.e. "scenery") was rotated around the stable platform. The rotating scenery also defined the shock sector. The classical and modified active place avoidance thus differed only by the presence/absence of inertial stimuli derived from passive rotation of the animal on the arena. The study has shown that these inertial stimuli are crucial for initial acquisition of the task. When the two versions were switched later in the training, animals with experience on the rotating arena performed well in the modified version, indicating that initial exposure to the classical version of the task was sufficient to provide the spatial associations necessary for task performance (Blahna et al. 2011). 


\section{Applications of the place avoidance tasks in combination with various experimental manipulations}

\section{Reversible and permanent ablations of brain regions}

\section{Hippocampus}

Experiments have demonstrated that the hippocampus is crucial for spatial navigation of laboratory animals (e.g. Morris et al. 1982). Animals with bilateral hippocampal lesions are not able to solve allothetic spatial tasks, such as the Morris water maze. Even partial hippocampal lesions impair retrieval of previously acquired spatial memory, suggesting distributed encoding of memory trace (Moser et al. 1995). Nevertheless, animals were still able to acquire the MWM with only approx. $25 \%$ of the hippocampus as long as the spared tissue was located at the septal pole of the hippocampus (Moser et al. 1995).

Cimadevilla et al. (2000c) investigated the influence of transient hippocampal inactivation on performance in the active place avoidance. Tetrodotoxin (TTX; 5ng in $1 \mu$ l saline), a sodium channel blocker, was administered bilaterally into the dorsal hippocampi via chronically implanted cannulae. This technique produces inactivation of a region of brain tissue within $\sim 1 \mathrm{~mm}$ from the infusion site for at least 4 hours (Zhuravin and Bures 1991, Klement et al. 2005). Infusions of TTX prevented retrieval of avoidance that was previously acquired during four days of training, while saline infusions did not. The hippocampal blockade by TTX resulted in retrieval disruption without affecting locomotion, suggesting a purely cognitive deficit. In addition, even unilateral hippocampal inactivation by TTX seriously impaired acquisition, consolidation and retrieval in the active place avoidance (Cimadevilla et al. 2001c). The most profound effect was observed during initial learning (acquisition), while retrieval was less impaired. TTX application after each session (in order to block consolidation of a memory trace) caused the mildest impairment, apparently due to partial consolidation that might have occurred just during the 20 -min acquisition session. These results are in striking contrast to the results from the Morris water maze, where TTX infusion into one hippocampus did not prevent the learning of a new escape location (Fenton and Bures 1993).

\section{Dentate gyrus}

The need for segregation of information in the active place avoidance suggests that the dentate gyrus may participate as a key player in navigation in dissociated reference frames. Czéh et al. (2001) studied spatial learning in rats with permanent damage to the dentate gyrus. Development of the dentate gyrus continues postnatally and x-ray irradiation of neonatal rats damages developing neurons. This results in a pronounced decrease in the numbers of granule cells in the dentate gyrus of adult rats. Their performance in the active place avoidance exhibited bimodal distribution with some rats being severely impaired, while others were able to learn the task. Nonetheless, even the irradiated rats that were capable of learning the task were impaired compared to controls. Moreover, they were also moderately impaired in arena-frame avoidance on a rotating arena in the dark (Arena+ avoidance). It appears that irradiation-induced disruption was more pronounced in the active place avoidance in light than in the arenabased task. No difference between irradiated and control rats was observed in a random foraging task with no navigational demands (Czéh et al. 2001). However, recent observation suggests that neuroinflammation associated with irradiation could significantly contribute to the observed deficits (A.A. Fenton; personal communication).

Suppression of adult hippocampal neurogenesis and effect on behavioral flexibility in mice

The relevance of adult-born neurons for normal hippocampal function is still only partially understood. Burghardt et al. (2012) examined the effect of two independent methods of ablation of adult-born hippocampal neurons (focal X-ray irradiation and genetic ablation of neural progenitors) in a mouse model, focusing on cognitive coordination and flexibility.

After mastering the active place avoidance task during initial training, the first group of mice had to adjust to a novel sector position, shifted by $180^{\circ}$, on the same arena (reversal). This shift introduced a conflict between the new situation and the already learned behavior. Although control mice coped with this situation well, animals with suppressed neurogenesis adopted less effective strategies and exhibited perseverative behavior. This contrasted with mice learning the same task, but in an entirely novel environment, where no impairment occurred in either group. Extinction of learned avoidance (when exposed to the arena without reinforcement) was 
also shown to be comparable between the groups. To probe a flexibility of a different kind, the authors took mice trained to avoid a room-frame sector, and added a new, arena-frame sector, making it a double-avoidance task. X-ray irradiated mice entered the new sector frequently, although they continued to avoid the original sector. When trained in the double-avoidance paradigm with two sectors present from the beginning, both groups managed to master it equally. Those results demonstrate that mice with suppressed are able to learn demanding spatial tasks with proficiency neurogenesis comparable to controls, but exhibit specific type of inflexibility, when change of the learned behavior is required. This was interpreted as an inability to segregate relevant memories form the irrelevant, as opposed to the ability to segregate spatial frames, which was preserved.

\section{Retrosplenial cortex}

The retrosplenial cortex (RSC) was another brain region studied in relation to the active place avoidance (Wesierska et al. 2009). This region is functionally connected with the hippocampal formation, as well as with many other cortical areas, and it was proposed that it may participate in the association of allothetic and idiothetic information (Aggleton 2010). Lesions of the RSC in rodents impair egocentric, but spare allothetic navigation (Cooper et al. 2001) as well as egocentric discrimination. These results indicate that the RSC may be critical for the interaction between different reference frames (Iaria et al. 2007). Rats with permanent bilateral lesions to the RSC by infusions of ibotenic acid show deficits very similar to those found in animals with unilateral hippocampal inactivations by TTX. They were impaired in conditions involving a strong reference frame conflict such as in the active place avoidance (Room+Arena- avoidance). If the conflict was attenuated by suppressing one set of cues, RSC-lesioned rats could avoid the sector efficiently. These findings support the idea that together with the hippocampus, the RSC is crucial for successful coordination of information from dissociated reference frames.

\section{Posterior parietal cortex}

Another brain structure tested for its involvement in the place avoidance tasks was the posterior parietal cortex (PPC). Effects of PPC lesions on navigation differed depending on the spatial task used. The most profound deficit was found in experiments focusing on egocentric navigation and utilization of proximal cues (Save and
Poucet 2000). Svoboda et al. (2008) investigated the effects of bilateral thermocoagulation lesions to the PPC in the active place avoidance on the rotating arena (Room+Arena- avoidance) and on the rotating arenaframe avoidance in darkness (Arena ${ }^{+}$avoidance). Rats were trained to forage for food pellets in the arena-based task, in which the arena rotated in darkness and the to-beavoided sector was defined according to the arena-frame reference. The authors hypothesized that animals would manage to solve the active place avoidance based on allothetic navigation and segregation of stimuli, but would fail in navigation in the arena-frame, requiring idiothetic navigation and association with proximal, substratal cues. Surprisingly, the lesioned animals solved both tasks as well as the controls. The PPC thus appears to be neither critical for cognitive coordination and allothetic navigation, nor for the idiothetic-proximal-cue variant of the place avoidance task (Svoboda et al. 2008).

\section{Neuropharmacological influences on active place avoidance}

The active place avoidance has also been used for neuropharmacological studies which selectively influenced different neurotransmitter systems by administering specific receptor agonists and antagonists. In the studies published so far (e.g. Stuchlik et al. 2004, Stuchlik and Vales 2005, Vales et al. 2006, Stuchlik et al. 2008), the drugs were administered systemically so that their action was not restricted to any particular brain region. Given the potential role of a cognitive coordination deficit in the cognitive symptoms of schizophrenia (Phillips and Silverstein 2003), the active place avoidance has been used to examine animal models of neuropsychiatric disorders (e.g. schizophrenia-like behavior in rats produced by systemic administration of non-competitive NMDA receptor antagonist MK-801; (Stuchlik et al. 2004, Stuchlik and Vales 2005, Vales et al. 2006) and possible beneficial effects of both established (Bubenikova-Valesova et al. 2008) and potential therapeutics (Vales et al. 2010, Vales et al. 2012). Illustrative examples of the arena tracks of the animals treated with various drugs are depicted in Figure 3. Panel A shows the performance of an intact rat, exhibiting efficient avoidance with activity only in the safe part of the arena. Panel B shows the trajectory of a hyperactive rat (here treated with $0.2 \mathrm{mg} / \mathrm{kg}$ MK-801, a non-competitive NMDA receptor blocker). Panel C shows the track of an animal impaired in avoidance, but still partly capable of an efficient escape reaction. 
NMDA receptor antagonists and an animal model of schizophrenia-like behavior induced by dizocilpine (MK-801)

According to one of the modern theories about the pathogenesis of schizophrenia, the cause of the disease may relate to neurodevelopment-related disruption of the glutamatergic neurotransmitter system (Kantrowitz and Javitt 2012). This hypothesis is based on the findings that systemic administration of noncompetitive antagonists of the N-methyl D-aspartate (NMDA) glutamate receptor such as ketamine or dizocilpine (MK-801) causes schizophrenia-like symptoms in healthy humans and exacerbate psychotic symptoms in schizophrenic patients (Newcomer and Krystal 2001). Administration of dizocilpine (MK-801) induces hyperlocomotion (Nilsson et al. 2001), general alterations of the animal's behavior, and cognitive deficits, which can be regarded as animal analogues of schizophrenic symptoms (schizophrenia-like behavior; Vales et al. 2006). Systemic administration of MK-801 to rats can be used as a model with predictive validity for testing the antipsychotic effects of drugs (e.g. Bubenikova-Valesova et al. 2008, Vales et al. 2010, 2012).

Several studies examined the effect of MK-801 on active place avoidance learning. Stuchlik et al. (2004) found no effect of $0.1 \mathrm{mg} / \mathrm{kg}$ of $\mathrm{MK}-801$ on place avoidance, although this dose was sufficient to impair water maze performance. A high dose of $0.2 \mathrm{mg} / \mathrm{kg}$ of MK-801 disrupted acquisition in both tasks. Stuchlik and Vales (2005) found that performance in a previously learned task in a familiar environment (retention) is disrupted by 0.2 , but not $0.15 \mathrm{mg} / \mathrm{kg}$, while reacquisition in a new environment is sensitive even to the lower dose.

Lobellova et al. (2013) tested the effect of low doses of MK-801 on reversal learning in the active place avoidance task and the water maze. They noted that the water maze reversal performance was influenced by doses of $0.1 \mathrm{mg} / \mathrm{kg}$ or higher, while mild deficits in place avoidance reversal occurred even at $0.08 \mathrm{mg} / \mathrm{kg}$. It can be concluded that the reversal learning in the active place avoidance task is more sensitive to MK-801, when compared to acquisition of the same task, or reversal learning in the water maze. All the above-mentioned results were obtained with Long-Evans rats, however, drug sensitivity can differ substantially in other rat strains. For example, Vales et al. (2006) found that Wistar rats are more sensitive to MK-801 than LongEvans in active place avoidance acquisition.
In an effort to test the therapeutic efficacy and validity of the model, Bubenikova-Valesova and colleagues (2008) investigated the effects of three compounds in an animal model of schizophrenia-like behavior induced by MK-801. Male adult albino Wistar rats were injected with $0.1 \mathrm{mg} / \mathrm{kg}$ of $\mathrm{MK}-801$, which impaired avoidance without increasing locomotion. Concurrently the rats received ritanserin (2.5 and $5 \mathrm{mg} / \mathrm{kg}$; a $5 \mathrm{HT}_{2 \mathrm{~A} / 2 \mathrm{C}}$ antagonist), risperidone $(0.1$ or $1 \mathrm{mg} / \mathrm{kg}$; an antipsychotic acting via blockade of D2 and $5 \mathrm{HT}_{2 \mathrm{~A} / 2 \mathrm{C}}$ receptors) and a classical antipsychotic haloperidol $(0.1$ and $1 \mathrm{mg} / \mathrm{kg} ;$ a D1 and D2 receptor antagonist). Haloperidol markedly decreased locomotion but did not alleviate the cognitive deficit induced by MK801. At both doses, both ritanserin and risperidone significantly reduced the dizocilpine-induced cognitive deficit in avoidance without affecting locomotion. The results also showed that substances with marked D2-like receptor antagonistic activity (such as haloperidol) induce a cognitive impairment even when given to naïve rats. It thus appears that $5 \mathrm{HT}_{2 \mathrm{~A} / 2 \mathrm{C}}$ receptor antagonism might be a more substantial factor in alleviating the cognitive deficit in a dizocilpine-induced animal model of psychosis-like symptoms than the blockade of D2-like receptors. Moreover, a recent study (Vales et al. 2010) has shown that the cognitive deficits in this animal model of schizophrenia can be alleviated with agonists and positive modulators of mGluR5 receptors. Both these findings support the predictive validity of this model of schizophrenia-like behaviors. Additionally, Vales et al. (2012) demonstrated that a novel neuroprotective substance, 3alpha-5beta-pregnanolone glutamate was able to reverse an MK-801-induced deficit in the active place avoidance task (with no foraging) without affecting locomotion.

Muscarinic, dopaminergic, and adrenergic receptor system manipulation

Many brain neurotransmitters differentially affect learning and memory including memories for space and navigational functions (Myhrer 2003). Muscarinic acetylcholine receptors (mAChR) are notoriously known for their involvement in spatial memory (as well as in other functions such as attention). Vales and Stuchlik (2005) studied the effect of scopolamine, an antagonist of a muscarinic subtype of acetylcholine receptors, on behavior in the active place avoidance. Scopolamine hydrobromide was administered intraperitoneally $(0.5,1$, and $2 \mathrm{mg} / \mathrm{kg}$ ). Animals were trained over four daily 
sessions with no injections and then subjected to a "reinforced retention" test (with the shock on) after scopolamine administration in a $5^{\text {th }}$ session in the same apparatus. Then they underwent five reacquisition sessions on a different arena with scopolamine injection before each session. This design was selected in order to elucidate the effect of mAChR blockade in rats previously exposed to the task rules ("intact pretraining") as cholinergic manipulations were documented to yield different results in pretrained rats (Beiko et al. 1997). The highest dose of scopolamine $(2 \mathrm{mg} / \mathrm{kg})$ increased locomotion and impaired avoidance in the retention session, as well as during the reacquisition training. Scopolamine $(1 \mathrm{mg} / \mathrm{kg})$ only impaired the retention test performance. It still remains unclear whether the scopolamine-induced active place avoidance deficit is due to an effect on place navigation per se or if the effect is mediated by drug-induced disruption of other aspects of the task (e.g. motivation, motor activity, behavioral flexibility etc.).

Dopamine and its receptors (divided into D1and D2-like superfamilies) are also involved in cognitive functions (Williams and Castner, 2006). Stuchlik and Vales (2006) investigated the effects of pharmacological manipulations of the dopaminergic system on the active place avoidance performance. The dopamine D1-like receptor antagonist (SCH23390; 0.02 and $0.05 \mathrm{mg} / \mathrm{kg}$ ) markedly impaired task performance and decreased locomotion. Low doses of the D1-like receptor agonist, A77636 (0.1 and $0.5 \mathrm{mg} / \mathrm{kg})$, had a facilitating effect; however, a subsequent study (Stuchlik 2007) showed that after pretraining to the task without drugs, no specific drug-induced changes were observed either in the retention test or during reacquisition on a different arena with new landmarks. The only observed effect was a motor deficit with $0.05 \mathrm{mg} / \mathrm{kg}$ of SCH 23390 during the retention test (Stuchlik 2007). Nonetheless, the systemic approach employed in the above studies precludes making any inferences about which specific brain structures may have been involved in the observed effects. Further studies involving site-specific injections of specific antagonists would be helpful in elucidating structure-specific modulation of place navigation in the active place avoidance task by dopamine receptors. The effects of dopamine D2-like receptor manipulations on spatial and locomotor parameters in the active place avoidance task showed a primary effect upon locomotion at doses lower than those necessary to disturb avoidance (Stuchlik et al. 2007a). A complementary study in the reference memory version of the MWM suggested that in other tasks, D2-like receptor antagonism may interfere with spatial navigation as well (Stuchlik et al. 2007b).

Noradrenaline receptors can also be involved in spatial memory and other cognitive functions, in addition to their known roles in vigilance, attention, fear, stress response etc. (Smythies 2005). Another series of experiments evaluated the effects of antagonism of all three major families of adrenergic receptors. Stuchlik and Vales (2008) examined the influence of an $\alpha 1$-antagonist, prazosin, and mainly presynaptic $\alpha 2$-antagonist, idazoxan, on the active place avoidance. The highest dose of prazosin $(4 \mathrm{mg} / \mathrm{kg})$, but not the lower doses $(0.5$ and $2 \mathrm{mg} / \mathrm{kg}$ ), decreased locomotor activity and negatively affected all measures of avoidance (non-specific deficit). Preliminary data from rats performing a non-locomotor operant task involving recognition of the position of a moving stimulus projected onto a computer screen (Levcik et al. 2013) suggested that motor impairment was indeed the primary cause of the observed deficit (Levcik et al. in press). Idazoxan similarly induced a combined deficit at $6 \mathrm{mg} / \mathrm{kg}$, but not at 1 or $3 \mathrm{mg} / \mathrm{kg}$, with decreased locomotion and an increased number of errors. The interaction between the $\alpha 1$ antagonist, prazosin, and the D2 antagonist, sulpiride, was studied by Stuchlik et al. (2008). A combination of sub-threshold doses, which alone did not elicit noticeable effects, caused severe impairment of locomotion and spatial avoidance. This suggests that such an interaction might indeed exist.

$\beta$-adrenoceptors, on the other hand, are widely recognized as important modulators of cognitive and memory functions. Potential amnestic effects of $\beta$ blockers (Cahill et al. 2000) may be useful in clinical psychiatry. Stuchlik et al. (2009) applied propranolol at four different doses before each of four acquisition sessions in the active place avoidance on the rotating arena. The low to medium doses ( 5 and $20 \mathrm{mg} / \mathrm{kg}$ ) had no effect, whereas the high dose $(30 \mathrm{mg} / \mathrm{kg})$ elicited overall sedation which disrupted the avoidance behavior. The intermediate dose $(25 \mathrm{mg} / \mathrm{kg})$ did not affect locomotion, but markedly increased the number of errors, suggesting a selective cognitive deficit. Concurrently, a pre-test application of 5 or $20 \mathrm{mg} / \mathrm{kg}$ of propranolol also failed to affect the time to the first entrance on the subsequent day, suggesting no effect on memory consolidation (or the absence of an effect on consolidation in this task per se).

Petrasek et al. (2010) also examined an effect of combined application of the alpha1-adrenoceptor antagonist prazosin (1 and $2 \mathrm{mg} / \mathrm{kg}$ ), and the beta blocker 
propranolol (5 and $20 \mathrm{mg} / \mathrm{kg}$ ). These doses were previously found not to impair active place avoidance when applied separately. However, after co-application of lower doses, the authors observed a cognitive deficit without changes in locomotion; locomotion was affected only by co-application of higher doses (Petrasek et al. 2010).

The effect of co-application of the D2-like receptor antagonist sulpiride and the beta-adrenergic blocker propranolol was studied by Prokopova et al. (2012). They found that doses which had no effect when administered separately induced notable impairment of performance when applied together, affecting both locomotion and cognitive parameters.

\section{Active place avoidance and the link between LTP and} memory

Pastalkova et al. (2006) used the active place avoidance to elucidate the molecular basis of long-term memory and its relationship to the maintenance phase of long-term potentiation (LTP) and specifically to the protein kinase Mzeta (PKMzeta). Manipulations interfering with LTP induction or maintenance also impair memory formation or consolidation, respectively. However, the direct link between long-lasting LTP and memory persistence has not been demonstrated. The maintenance phase of late-LTP critically depends on the activity of PKMzeta, an atypical form of protein kinase $\mathrm{C}$ (for demonstration of the principle on the tissue level, see Serrano et al. (2005). Administration of an inhibitor of PKMzeta, a synthetic peptide known as "ZIP" (zeta inhibiting peptide), affected neither LTP induction nor the early-LTP phase. While it did not damage normal neuronal function, it did abolish the maintenance phase of late LTP. In the study of Pastalkova et al. (2006), rats acquired active place avoidance and the next day the ZIP peptide was injected into both hippocampi. Testing in an extinction condition (with the shock inactivated) two hours after the injection revealed that ZIP abolished avoidance, while control animals avoided normally. This demonstrated the "erasure" of the memory trace by application of ZIP. However, blockade of PKMzeta did not affect new learning. Another experiment was conducted, in which rats received ZIP injections 24 hours after learning, but were not tested earlier than a week after ZIP administration. By this time, the inhibitor had already been metabolized and thus no longer present in their brains; however, the rats were still unable to avoid the shock sector efficiently. ZIP administration eliminated avoidance memories even as old as one month, suggesting that PKMzeta might indeed be the "memory molecule". The effect of the ZIP peptide differed from that of staurosporine, which only blocked LTP induction but not maintenance, and elicited anterograde, instead of retrograde amnesia. Hippocampal inactivation by TTX also did not affect consolidated memory traces; it only blocked new learning, initial memory consolidation and memory retrieval, but not its retention. These promising results were later corroborated by Serrano and colleagues (2008) in other memory domains. However, a recent study by Volk et al. (2013) generated transgenic rats lacking PKMzeta by constitutive and inducible knockout and did not find any deterioration of either LTP or spatial memory. Moreover, application of ZIP to these transgenic animals abolished LTP, suggesting that there might be at least one additional substrate for this oligopeptide than PKMzeta. In light of these results, the task for future studies is to explore novel substrates of ZIP in order to corroborate the hypothesized link between LTP and spatial memory.

Neonatal ventral hippocampus lesion model of schizophrenia

The neonatal ventral hippocampus lesion in the rat (NVHL) (Chambers et al. 1996, Tseng et al. 2009) constitutes an important and well-studied neurodevelopmental model of schizophrenia. Lee et al. (2012) studied the effect of the neonatal (postnatal day 7) lesion on performance of adult rats in various tasks on a rotating arena, and tested the hypothesis that the impairments could be alleviated by cognitive training during adolescence, i.e. before the onset of schizophrenia-like symptoms. Untreated adult NVHL rats exhibited slower learning in the active place avoidance task, and a severe impairment after reversal, whereas their retention (long-term memory) was normal. When the conflict of reference frames was alleviated by masking arena-frame olfactory cues by shallow water, NVHL rats learned the task without difficulty, suggesting rather specific impairment of cognitive control. Adolescent NVHL rats exhibited no impairment in the active place avoidance task, suggesting that the schizophrenia-like symptoms were absent on postnatal day 35. The training during adolescence had a profound impact on their further development, however. When the same rats were tested again as adults, their cognitive impairments were completely abolished. This behavioral benefit included not only the active place avoidance and 
reversal therein, but also reversal learning in the T-maze. Therefore, it extended well beyond the adolescent experience and was not task specific. NVHL rats that were only exposed to the arena (without learning any task) during adolescence, performed poorly when tested as adults, confirming that the exposure to a novel environment during the sensitive period is not sufficient to reverse the effect of NVHL. Another important finding of this study concerns local field potentials in the hippocampus. Interhippocampal synchrony was found to correlate with performance in the active place avoidance task and was facilitated by cognitive training during adolescence.

\section{Traumatic brain injury}

Avoidance tasks on a dry arena have the great advantage of being very flexible. A battery of test variants differing in cognitive demands can be easily designed using a single apparatus, enabling not only detection of impairment, but also allowing for the assessment of it's nature and severity. This approach was applied by Abdel Baki et al. $(2009,2010)$ in studies of traumatic brain injury and its possible treatments. The authors applied four different tasks: Spontaneous open field exploration, passive place avoidance, active place avoidance and active avoidance reversal (conflict). The rats tested were those either after mild or moderate traumatic brain injury, induced by the controlled cortical impact method, or sham-operated controls. In the first two tasks, all rats performed comparably. During the active place avoidance training phase, rats with moderate brain injury exhibited marked impairment, whereas mild injury had no effect. When the sector position was reversed, however, even the mildly injured animals failed to learn. This suggests that the ability to distinguish relevant memories or learned responses from the irrelevant when they are conflicted during reversal training is more sensitive to traumatic damage than the ability to separate information from relevant and irrelevant spatial frames.

Those findings were applied by Abdel Baki et al. (2010), who compared the efficiency of different drugs and drug combinations which are proposed as neuroprotective treatment for moderate traumatic brain injury in a similar experimental setting. This particular study also illustrates the importance of the training schedule used for the behavioral training. A "massed" active place avoidance training schedule, with six 10-min trials during a single day, separated by 10 -min inter-trial intervals, was used in the initial screening. Both minocycline and the combination of minocycline with $\mathrm{N}$ acetylcysteine succeeded in alleviating the impairment observed after brain injury, although not in restoring the cognitive abilities to the control level. Subsequently, another experimental protocol, the so-called "spaced" training, was applied. Here, the animals were trained for 15 days with a single 20-min session each day. This setup was chosen to examine possible impairments of longterm memory. For this protocol, only the combination of minocycline with $\mathrm{N}$-acetylcysteine was effective, while minocycline alone failed to exhibit any benefit.

\section{Other interventions}

The place avoidance has also been used to evaluate other experimental manipulations. Wesierska et al. (2006) used the advantage of the rotating arena tasks to test rats with experimentally-induced toxic liver failure. In human patients, liver insufficiency may lead to a hepatic encephalopathy, in which increased ammonium levels may cause motor and cognitive deficits. Rats were able to learn the active place avoidance after intoxication with thioacetamide - i.e., they exhibited intact spatial navigation and cognitive coordination. Nevertheless, they were severely impaired during subsequent training in the arena-frame task in the dark, in which they were required to use the previously irrelevant stimuli. The intoxicated animals thus exhibited lower cognitive flexibility than the control rats. Therefore, the authors proposed the use of cognitive flexibility tests in the human diagnosis of mild forms of hepatic encephalopathy.

The active place avoidance task has also been recently used to study the effects of transcranial direct current stimulation on rat performance in the APAAT variant (Dockery et al. 2011). Transcranial direct current stimulation (tDCS) is a method used in humans and animals to transiently alter neuronal excitability via weak direct currents. It is useful to study the causal role of structures within neural networks on specified functions (Stagg and Nitsche 2011). In humans it is already employed for clinical treatment of depression, stroke and other neuropsychiatric disorders (Kalu et al. 2012), however systematic evaluation of tDCS effects on the underlying cellular mechanisms is lacking which calls for development of appropriate animal models. This study focused on the role of frontal activity in performance in an APAAT task, described by Dockery and Wesierska (2010). In rats, tDCS was successfully used to manipulate visuospatial working memory and experience-dependent 
skill learning in the APAAT. The results suggest cumulative benefits of cathodal tDCS on rat task performance following $30 \mathrm{~min}$ of $200 \mu \mathrm{A}$ current over the frontal cortex. The effects were polarity specific as neither the rats undergoing anodal tDCS nor the control rats expressed boosted performance. The treatment was applied offline during the training period; therefore the benefits of tDCS on later performance in the APAAT were ascribed to a cumulative function of optimized neuronal activity during acquisition related to neural plasticity. Since cathodal tDCS is thought to reduce neuronal excitability, while anodal tDCS is thought to increase it, the authors proposed that cathodal tDCS may reduce superfluous neuronal activity in the frontal cortex in response to novel conditions and task difficulty, thereby resulting over the long-term in higher avoidance efficiency (Dockery et al. 2011). Such underlying mechanisms proposed to be involved in the effects of tDCS of the frontal cortex on memory in the APAAT are further explored in a work by Dockery (2013).

Carr et al. (2011) used the place avoidance paradigm for a study of postoperative cognitive dysfunction. The rats were trained in the active place avoidance paradigm, and then underwent two hours of isoflurane anesthesia. On days 1, 3 and 7 after the treatment they were tested in the apparatus again, to probe their retention of the learned behavior. The rats that had undergone the anesthesia exhibited lower locomotion during the test sessions, relative to control (untreated) animals, and exhibited signs of mild cognitive impairment.

\section{Concluding remarks}

Place avoidance tasks represent effective tools for the assessment of spatial learning and navigation in rodents. The place avoidance paradigm allows for precise control of the sources and modalities of spatial information available to the experimental animals. It therefore facilitates individual component analysis of animal navigation. The active place avoidance has become a useful spatial task, beneficial for the evaluation of experimental interventions (such as brain lesions or drugs) on cognitive and non-cognitive aspects of rodent spatial behavior, investigations of animal models of psychiatric disorders and brain injuries or unit recordings from behaving animals. In active place avoidance, efficient navigation depends on allothetic spatial learning, presumably with both short-term and long-term memory components. Moreover, this task requires the segregation of relevant and irrelevant information and the construction of separate but interacting spatial representations in two dissociated spatial reference frames (arena- and room-frame). This paradigm taxes different aspects of place navigation when compared to the Morris water maze and other commonly used tasks, and thus it substantially enriches the spectrum of cognitive functions that can be assessed in animals. Although a relatively novel method, the active place avoidance is already employed by several laboratories pursuing interesting and influential questions related to spatial cognition, learning and memory. As a paradigm it holds great promise to bring a deeper understanding of cognitive functions, and helps to elucidate the mechanisms underlying various neuropsychiatric disorders.

\section{Conflict of Interest}

There is no conflict of interest.

\section{Acknowledgements}

This study was supported by GACR P304/12/G069 and P303/10/P191, IGA MZ CR NT13386 and ASCR project M200111204, by Institutional support RVO:67985823 and AV0Z50110509 and grant MNiSW 8165/B/P01/2011/40. We thank Jan Bures and André Antonio Fenton for critical comments on early versions of the manuscript.

\section{References}

ABDEL BAKI SG, KAO HY, KELEMEN E, FENTON AA, BERGOLD PJ: A hierarchy of neurobehavioral tasks discriminates between mild and moderate brain injury in rats. Brain Res 1280: 98-106, 2009.

ABDEL BAKI SG, SCHWAB B, HABER M, FENTON AA, BERGOLD PJ: Minocycline synergizes with Nacetylcysteine and improves cognition and memory following traumatic brain injury in rats. PLoS One 5 : e12490, 2010.

AGGLETON JP: Understanding retrosplenial amnesia: insights from animal studies. Neuropsychologia 48: 2328-2338, 2010. 
BARNES CA: Memory deficits associated with senescence: a neurophysiological and behavioral study in the rat. J Comp Physiol Psychol 93: 74-104, 1979.

BEIKO J, CANDUSSO L, CAIN DP: The effect of nonspatial water maze pretraining in rats subjected to serotonin depletion and muscarinic receptor antagonism: a detailed behavioural assessment of spatial performance. Behav Brain Res 88: 201-211, 1997.

BLAHNA K, SVOBODA J, TELENSKY P, KLEMENT D: Inertial stimuli generated by arena rotation are important for acquisition of the active place avoidance task. Behav Brain Res 216: 207-213, 2011.

BUBENIKOVA-VALESOVA V, STUCHLIK A, SVOBODA J, BURES J, VALES K: Risperidone and ritanserin but not haloperidol block effect of dizocilpine on the active allothetic place avoidance task. Proc Natl Acad Sci USA 105: 1061-1066, 2008.

BUHUSI CV, MECK WH: What makes us tick? Functional and neural mechanisms of interval timing. Nat Rev Neurosci 6: 755-765, 2005.

BURES J, FENTON AA, KAMINSKY YU, ZINYUK L: Place cells and place navigation. Proc Natl Acad Sci USA 94: 343-350, 1997a.

BURES J, FENTON AA, KAMINSKY YU, ROSSIER J, SACCHETTI B, ZINYUK L: Dissociation of exteroceptive and idiothetic orientation cues: effect on hippocampal place cells and place navigation. Phil Trans $R$ Soc Lond 352: 1515-1524, 1997b.

BURES J, FENTON AA, KAMINSKY YU, WESIERSKA M, ZAHALKA A: Rodent navigation after dissociation of the allocentric and idiothetic representations of space. Neuropharmacology 37: 689-699, 1998.

BURGHARDT NS, PARK EH, HEN R, FENTON AA: Adult-born hippocampal neurons promote cognitive flexibility in mice. Hippocampus 22: 1795-1808, 2012.

CAHILL L, PHAM CA, SETLOW B: Impaired memory consolidation in rats produced with $\beta$-adrenergic blockade. Neurobiol Learn Mem 74: 259-266, 2000.

CARR ZJ, TORJMAN MC, MANU K, DY G, GOLDBERG ME: Spatial memory using active allothetic place avoidance in adult rats after isoflurane anesthesia: a potential model for postoperative cognitive dysfunction. J Neurosurg Anesthesiol 23: 138-145, 2011.

CHAMBERS RA, MOORE J, MCEVOY JP, LEVIN ED: Cognitive effects of neonatal hippocampal lesions in a rat model of schizophrenia. Neuropsychopharmacology 15: 587-594, 1996.

CIMADEVILLA JM, FENTON AA, BURES J: Continuous place avoidance task reveals differences in spatial navigation in male and female rats. Behav Brain Res 107: 161-169, 2000a.

CIMADEVILLA JM, KAMINSKY YU, FENTON A, BURES J: Passive and active place avoidance as a tool of spatial memory research in rats. $J$ Neurosci Methods 102: 155-164, 2000 b.

CIMADEVILLA JM, FENTON AA, BURES J: Functional inactivation of dorsal hippocampus impairs active place avoidance in rats. Neurosci Lett 285: 53-56, 2000c.

CIMADEVILLA JM, FENTON AA, BURES J: Transient sex differences in the between-sessions but not in the withinsession memory underlying an active place avoidance task in weanling rats. Behav Neurosci 115: 695-703, 2001a.

CIMADEVILLA JM, FENTON AA, BURES J: New spatial cognition tests for mice: passive place avoidance on stable and active place avoidance on rotating arenas. Brain Res Bull 54: 559-563, $2001 \mathrm{~b}$.

CIMADEVILLA JM, WESIERSKA M, FENTON AA, BURES J: Inactivating one hippocampus impairs avoidance of a stable room-defined place during dissociation of arena cues from room cues by rotation of the arena. Proc Natl Acad Sci USA 98: 3531-3536, 2001c.

COOPER BG, MANKA TF, MIZUMORI SJ: Finding your way in the dark: the retrosplenial cortex contributes to spatial memory and navigation without visual cues. Behav Neurosci 115: 1012-1028, 2001.

CZEH B, STUCHLIK A, WESIERSKA M, CIMADEVILLA JM, POKORNY J, SERESS L, BURES J: Effect of neonatal dentate gyrus lesion on allothetic and idiothetic navigation in rats. Neurobiol Learn Mem 75: 190-213, 2001. 
DOCKERY CA: The human experiment: how we won't win the rat race. what can we learn from brain stimulation in humans and rats about enhancing the functional neurobiology of higher cognitive functions? In: Cognitive Enhancement. An Interdisciplinary Perspective Series (Trends in Augmentation of Human Performance). E HILDE, AG FRANKE (eds), Springer, Dordrecht, 2013, pp 73-91.

DOCKERY CA, WESIERSKA MJ: A spatial paradigm, the allothetic place avoidance alternation task, for testing visuospatial working memory and skill learning in rats. $J$ Neurosci Methods 191: 215-221, 2010.

DOCKERY CA, LIEBETANZ D, BIRBAUMER N, MALINOWSKA M, WESIERSKA MJ: Cumulative benefits of frontal transcranial direct current stimulation on visuospatial working memory training and skill learning in rats. Neurobiol Learn Mem 96: 452-460, 2011.

ELLENBROEK BA, COOLS AR: Animal models with construct validity for schizophrenia. Behav Pharmacol 1: 469490, 1990.

FENTON AA, BURES J: Place navigation in rats with unilateral tetrodotoxin inactivation of the dorsal hippocampus: place but not procedural learning can be lateralized to one hippocampus. Behav Neurosci 107: 552-564, 1993.

FENTON AA, WESIERSKA M, KAMINSKY YU, BURES J: Both here and there: Simultaneous expression of autonomous spatial memories in rats. Proc Natl Acad Sci USA 95: 11493-11498, 1998.

IARIA G, CHEN JK, GUARIGLIA C, PTITO A, PETRIDES M: Retrosplenial and hippocampal brain regions in human navigation: complementary functional contributions to the formation and use of cognitive maps. Eur $J$ Neurosci 25: 890-899, 2007.

KALU UG, SEXTON CE, LOO CK, EBMEIER KP: Transcranial direct current stimulation in the treatment of major depression: a meta-analysis. Psychol Med 12: 1-10, 2012.

KANTROWITZ J, JAVITT DC: Glutamatergic transmission in schizophrenia: from basic research to clinical practice. Curr Opin Psychiatry 25: 96-102, 2012.

KELEMEN E, FENTON AA: Dynamic grouping of hippocampal neural activity during cognitive control of two spatial frames. PLoS Biol 8: e1000403, 2010.

KLEMENT D, PASTALKOVA E, FENTON AA: Tetrodotoxin infusions into the dorsal hippocampus block nonlocomotor place recognition. Hippocampus 15: 460-471, 2005.

KONORSKI J: Conditioned Reflexes and Neuron Organization. Cambridge Univ. Press, Cambridge, 1948.

KUBIK S, FENTON AA: Behavioral evidence that segregation and representation are dissociable hippocampal functions. J Neurosci 25: 9205-9212, 2005.

LEE H, DVORAK D, KAO HY, DUFFY ÁM, SCHARFMAN HE, FENTON AA: Early cognitive experience prevents adult deficits in a neurodevelopmental schizophrenia model. Neuron 75: 714-724, 2012.

LEVCIK D, NEKOVAROVA T, STUCHLIK A, KLEMENT D: Rats use hippocampus to recognize positions of objects located in an inaccessible space. Hippocampus 23: 153-161, 2013.

LOBELlOVA V, ENTLEROVA M, SVOJANOVSKA B, HATALOVA H, PROKOPOVA I, PETRASEK T, VALES K, KUBIK S, FAJNEROVA I, STUCHLIK A: Two learning tasks provide evidence for disrupted behavioural flexibility in an animal model of schizophrenia-like behaviour induced by acute MK-801: a dose-response study. Behav Brain Res 246: 55-62, 2013.

MORRIS RGM: Spatial localization does not require the presence of local cues. Learn Motiv 12: 239-261, 1981.

MORRIS RGM, GARRUD P, RAWLINGS J, O'KEEFE J: Place navigation impaired in rats with hippocampal lesions. Nature 297: 681-683, 1982.

MOSER MB, MOSER EI, FORREST E, ANDERSEN P, MORRIS RGM: Spatial learning with a minislab in the dorsal hippocampus. Proc Natl Acad Sci USA 92: 9697-9701, 1995.

MULLER R: A quarter of a century of place cells. Neuron 17: 813-822, 1996.

MYHRER T: Neurotransmitter systems involved in learning and memory in the rat: a meta-analysis based on studies of four behavioral tasks. Brain Res Rev 41: 268-287, 2003.

NEWCOMER JW, KRYSTAL JH: NMDA receptor regulation of memory and behavior in humans. Hippocampus 11: 529-542, 2001.

NILSSON M, WATERS S, WATERS N, CARLSSON A, CARLSSON ML: A behavioural pattern analysis of hypoglutamatergic mice - effects of four different antipsychotic agents. J Neural Transm 108: 1181-1196, 2001. 
O'KEEFE J, NADEL L: Hippocampus as a Cognitive Map. Clarendon Press, London, 1978.

OLYPHER AV, KLEMENT D, FENTON AA: Cognitive disorganization in hippocampus: a physiological model of the disorganization in psychosis. J Neurosci 26: 158-168, 2006.

PASTALKOVA E, SERRANO P, PINKHASOVA D, WALLACE E, FENTON AA, SACKTOR TC: Storage of spatial information by the maintenance mechanism of LTP. Science 25: 1141-1144, 2006.

PETRASEK T, DOULAMES V, PROKOPOVA I, VALES K, STUCHLIK A: Combined administration of alpha1adrenoceptor antagonist prazosin and beta-blocker propranolol impairs spatial avoidance learning on a dry arena. Behav Brain Res 208: 402-407, 2010.

PHILLIPS WA, SILVERSTEIN SM: Convergence of biological and psychological perspectives on cognitive coordination in schizophrenia. Behav Brain Sci 26: 65-82, 2003.

PROKOPOVA I, BAHNIK S, DOULAMES V, VALES K, PETRASEK T, SVOBODA J, STUCHLIK A: Synergistic effects of dopamine D2-like receptor antagonist sulpiride and beta-blocker propranolol on learning in the Carousel maze, a dry-land spatial navigation task. Pharmacol Biochem Behav 102: 151-156, 2012.

SAVE E, POUCET B: Hippocampal-parietal cortical interactions in spatial cognition. Hippocampus 10: 491-499, 2000.

SERRANO P, YAO Y, SACKTOR TC: Persistent phosphorylation by protein kinase Mzeta maintains late-phase longterm potentiation. J Neurosci 25: 1979-1984, 2005.

SERRANO P, FRIEDMAN EL, KENNEY J, TAUBENFELD SM, ZIMMERMAN JM, HANNA J, ALBERINI C, KELLEY AE, MAREN S, RUDY JW, YIN JC, SACKTOR TC, FENTON AA: PKMzeta maintains spatial, instrumental, and classically conditioned long-term memories. PLoS Biol 6: 2698-2706, 2008.

SMYTHIES J: Section III. The norepinephrine system. Int Rev Neurobiol 64: 173-211, 2005.

STAGG CJ, NITSCHE MA: Physiological basis of transcranial direct current stimulation. Neuroscientist 17: 37-53, 2011.

STUCHLIK A: Further study of the effects of dopaminergic D1 drugs on place avoidance behavior using pretraining: some negative evidence. Behav Brain Res 178: 47-52, 2007.

STUCHLIK A, BURES J: Relative contribution of allothetic and idiothetic navigation to place avoidance on stable and rotating arenas in darkness. Behav Brain Res 128: 179-188, 2002.

STUCHLIK A, VALES K: Systemic administration of MK-801, a non-competitive NMDA-receptor antagonist, elicits a behavioural deficit of rats in the Active Allothetic Place Avoidance (AAPA) task irrespectively of their intact spatial pretraining. Behav Brain Res 159: 163-171, 2005.

STUCHLIK A, VALES K: Effect of dopamine D1 receptor antagonist SCH23390 and D1 agonist A77636 on active allothetic place avoidance, a spatial cognition task. Behav Brain Res 172: 250-255, 2006.

STUCHLIK A, VALES K: Role of alpha1- and alpha2-adrenoceptors in the regulation of locomotion and spatial behavior in the active place avoidance task: a dose-response study. Neurosci Lett 433: 235-240, 2008.

STUCHLIK A, FENTON AA, BURES J: Substratal idiothetic navigation of rats is impaired by removal or devaluation of extramaze and intramaze cues. Proc Natl Acad Sci USA 98: 3537-3542, 2001.

STUCHLIK A, REZACOVA L, VALES K, BUBENIKOVA V, KUBIK S: Application of a novel Active Allothetic Place Avoidance task (AAPA) in testing a pharmacological model of psychosis in rats: comparison with the Morris water maze. Neurosci Lett 366: 162-166, 2004.

STUCHLIK A, REHAKOVA L, RAMBOUSEK L, SVOBODA J, VALES K: Manipulation of D2 receptors with quinpirole and sulpiride affects locomotor activity before spatial behavior of rats in an active place avoidance task. Neurosci Res 58: 133-139, 2007a.

STUCHLIK A, REHAKOVA L, TELENSKY P, VALES K: Morris water maze learning in Long-Evans rats is differentially affected by blockade of D1-like and D2-like dopamine receptors. Neurosci Lett 422: 169-174, $2007 \mathrm{~b}$

STUCHLIK A, PETRÁSEK T, VALES K: Dopamine D2 receptors and alpha1-adrenoceptors synergistically modulate locomotion and behavior of rats in a place avoidance task. Behav Brain Res 189: 139-144, 2008.

STUCHLIK A, PETRÁSEK T, VALES K: A dose-response study of effects of pre-test administration of betaadrenergic antagonist propranolol on the learning of active place avoidance, a spatial cognition task, in rats. Behav Brain Res 200: 144-149, 2009. 
SVOBODA J, TELENSKY P, BLAHNA K, ZACH P, BURES J, STUCHLIK A: Lesion of posterior parietal cortex in rats does not disrupt place avoidance based on either distal or proximal orienting cues. Neurosci Lett 445: 7377, 2008.

TSENG KY, CHAMBERS RA, LIPSKA BK: The neonatal ventral hippocampal lesion as a heuristic neurodevelopmental model of schizophrenia. Behav Brain Res 204: 295-305, 2009.

VALES K, STUCHLIK A: Central muscarinic blockade interferes with retrieval and reacquisition of active allothetic place avoidance despite spatial pretraining. Behav Brain Res 161: 238-244, 2005.

VALES K, BUBENIKOVA-VALESOVA V, KLEMENT D, STUCHLIK A: Analysis of sensitivity to MK-801 treatment in a novel active allothetic place avoidance task and in the working memory version of the Morris water maze reveals differences between Long-Evans and Wistar rats. Neurosci Res 55: 383-388, 2006.

VALES K, SVOBODA J, BENKOVICOVA K, BUBENIKOVA-VALESOVA V, STUCHLIK A: The difference in effect of mGlu2/3 and mGlu5 receptor agonists on cognitive impairment induced by MK-801. Eur $J$ Pharmacol 639: 91-98, 2010.

VALES K, RAMBOUSEK L, HOLUBOVA K, SVOBODA J, BUBENIKOVA-VALESOVA V, CHODOUNSKA H, VYKLICKY L, STUCHLIK A: 3 $\alpha 5 \beta$-Pregnanolone glutamate, a use-dependent NMDA antagonist, reversed spatial learning deficit in an animal model of schizophrenia. Behav Brain Res 235: 82-88, 2012.

VOLK LJ, BACHMAN JL, JOHNSON R, YU Y, HUGANIR RL: PKM- $\zeta$ is not required for hippocampal synaptic plasticity, learning and memory. Nature 493: 420-423, 2013.

WESIERSKA M, DOCKERY C, FENTON AA: Beyond memory, navigation, and inhibition: behavioral evidence for hippocampus-dependent cognitive coordination in the rat. J Neurosci 25: 2413-2419, 2005.

WESIERSKA M, KLINOWSKA HD, ADAMSKA I, FRESKO I, SADOWSKA J, ALBRECHT J: Cognitive flexibility but not cognitive coordination is affected in rats with toxic liver failure. Behav Brain Res 171: 70-77, 2006.

WESIERSKA M, ADAMSKA I, MALINOWSKA M: Retrosplenial cortex lesion affected segregation of spatial information in place avoidance task in the rat. Neurobiol Learn Mem 91: 41-49, 2009.

WILLIAMS GV, CASTNER SA: Under the curve: critical issues for elucidating D1 receptor function in working memory. Neuroscience 139: 263-276, 2006.

ZHURAVIN IA, BURES J: Extent of the tetrodotoxin induced blockade examined by pupillary paralysis elicited by intracerebral injection of the drug. Exp Brain Res 83: 687-690, 1991. 